\title{
Charged Excitons in Self-assembled Quantum Dots
}

\author{
R. J. Warburton ${ }^{1}$, B. Urbaszek ${ }^{1}$, E. J. McGhee ${ }^{1}$, C. Schulhauser ${ }^{2}$, A. Högele ${ }^{2}$,

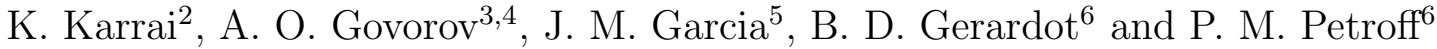 \\ ${ }^{1}$ Department of Physics, Heriot-Watt University, Edinburgh, UK \\ ${ }^{2}$ Sektion Physik, Ludwig-Maximilians-Universität, Munich, Germany \\ ${ }^{3}$ Department of Physics and Astronomy, Ohio University, Athens, USA \\ ${ }^{4}$ Institute of Semiconductor Physics, 630090 Novosibirsk, Russia \\ ${ }^{5}$ Instituto de Microelectrónica, Madrid, Spain \\ ${ }^{6}$ Materials Department, University of California, Santa Barbara, USA
}

\section{ABSTRACT}

We have succeeded in generating highly charged excitons in InAs self-assembled quantum dots by embedding the dots in a field-effect heterostructure. We discover an excitonic Coulomb blockage: over large regions of gate voltage, the exciton charge remains constant. We present here a summary of the emission properties of the charged excitons.

\section{INTRODUCTION}

An exciton is the elementary excitation in a semiconductor consisting of an electron and a valence level hole, bound together by the Coulomb potential. The exciton decays by photon emission when the electron recombines with the hole, which is a strong optical process because the exciton and vacuum states are connected by the electric dipole operator. The exciton ionization energy can be increased in a quantum well relative to that in bulk material by the quantum confinement. An increase up to about $10 \mathrm{meV}$ from a bulk value of about $4 \mathrm{meV}$ is possible in a GaAs quantum well [1]. A charged exciton, consisting of two electrons and a hole, is just bound in a quantum well. More highly-charged excitons are however unbound and do not exist. This state-of-affairs changes in a self-assembled quantum dot where in addition to a vertical confinement, there is also a lateral confinement arising from the nanometer-sized dot. In this case, the exciton binding energy (defined as the energy required to separate an electron and a hole and place them in identical but well separated quantum dots) increases to, say, $30 \mathrm{meV}$ for an InAs/GaAs quantum dot [2]. In fact, the quantum confinement is so strong in these systems that it is more accurate to refer to a highly-confined electron-hole pair rather than an exciton. The strong confinement in a self-assembled quantum dot opens up the possibility for the first time of investigating highly-charged excitons because even excitons with several excess electrons are stable. We have shown how it is possible to generate these highly-charged excitons in a single self-assembled quantum dots by embedding the quantum dots in an appropriate field-effect heterostructure [3]. We label the excitons with $\mathrm{X}^{n-}$ where $n$ is the excess charge. We have detected photoluminescence (PL) from the neutral exciton $\mathrm{X}^{0}$ up to the quadruply-charged exciton $\mathrm{X}^{4-}$. Each time an exciton gains an additional electron, its PL red-shifts through the Coulomb interaction, with the jumps in wavelength revealing a shell structure. Furthermore, for the $\mathrm{X}^{2-}$ and $\mathrm{X}^{2-}$, splittings arise, related to electron-hole exchange effects in the initial states, and electron-electron exchange effects in the final states. In this paper, we summarize the behavior of charged excitons in self-assembled quantum dots. 


\section{HETEROSTRUCTURE DESIGN}

In our field-effect heterostructure, the InAs quantum dots are self-assembled on undoped GaAs, $25 \mathrm{~nm}$ above an $\mathrm{n}^{+}$-region, the back contact. The dots are capped with a total of $150 \mathrm{~nm}$ of GaAs/AlAs. We make contacts to the $\mathrm{n}^{+}$-layer and deposit a semi-transparent $\mathrm{NiCr}$ gate on the sample surface. A voltage $V_{g}$ is applied to the gate, with the back contact grounded. At large negative $V_{g}$, the ground state of a particular dot lies above the Fermi energy and so the dot is unoccupied at low temperature. At a more positive $V_{g}$, there is a resonance between the dot's ground state and the Fermi level in the back contact allowing an electron to tunnel in and out. A further increase in $V_{g}$ traps an electron on the dot, and a Coulomb blockade results: a significantly larger voltage is required to induce tunneling of a second electron from the back contact into the dot. The Coulomb blockade is particularly pronounced because the quantum dots are so small, resulting in large Coulomb energies. We perform PL measurements on individual dots at $4.2 \mathrm{~K}$, recording the PL as a function of $V_{g}$. In all the experiments, the excitation (between 820 and $850 \mathrm{~nm}$ wavelength) is kept low enough that the biexciton-related features are much weaker than the exciton-related features.

\section{NEUTRAL AND SINGLY-CHARGED EXCITONS}

A color scale plot of the PL versus $V_{g}$ is shown in Fig. for a particular quantum dot. It can be seen that there are large regions of voltage where PL from only one charge state is observed. In the transition regions, there is a transfer of intensity from one charge state to the next; the transition is not completely abrupt because of thermal occupation of the higher energy state. In other words, an exciton in this sample always has sufficient time to find the charge state with the minimum energy before recombination occurs. This can be termed an excitonic Coulomb blockade. At large and negative $V_{g}$, the lowest energy state is an empty dot and for this reason there is no PL. At less negative $V_{g}$ however, the exciton is stable, and we resolve a sharp PL line typical of the neutral exciton in a single dot at low temperature. At $\sim-0.5 \mathrm{~V}$, the PL from the dot in Fig. jumps to the red. This is the transition from a neutral exciton to a charged exciton: the voltage is positive enough that the exciton with the lowest energy is $\mathrm{X}^{1-}$, not $\mathrm{X}^{0}$. The red-shift occurs because, on adding an electron to a neutral exciton, the Coulomb attraction of the electron and hole is larger than the Coulomb repulsion of the two electrons. This is a consequence of the fact that the hole has a wave function with a smaller lateral extent than the electron [2]. An obvious feature in Fig. is that the $\mathrm{X}^{1-}$ exists over a larger range in $V_{g}$ than the $\mathrm{X}^{0}$. The reason for this is that the width of the $\mathrm{X}^{0}$ plateau is determined by a Coulomb energy, but the $\mathrm{X}^{1-}$ plateau width is determined not only by the Coulomb energy but also by the quantization energy: in order to form an $\mathrm{X}^{2-}$, an electron must tunnel into the excited state.

\section{DOUBLY-CHARGED EXCITONS: EXCHANGE INTERACTIONS}

For the $\mathrm{X}^{2-}$, the PL exhibits not a single line, but, at first glance, two peaks separated by $\sim 5 \mathrm{meV}$ (Fig.s and ). The origin of this splitting is an exchange interaction in the final state. The initial state has electron spin $S=\frac{1}{2}$; the final state has spin $S=1$ or $S=0$. The possible configurations, labelling the electron ground state orbital $s$ and the first excited orbital $p$ in analogy to atomic physics, are shown in Fig. . The final states are split by twice the exchange energy between an $s$ and a $p$ electron. A very marked feature of the PL is that the higher energy emission into the $S=1$ state has a sharp line, whereas the emission into 


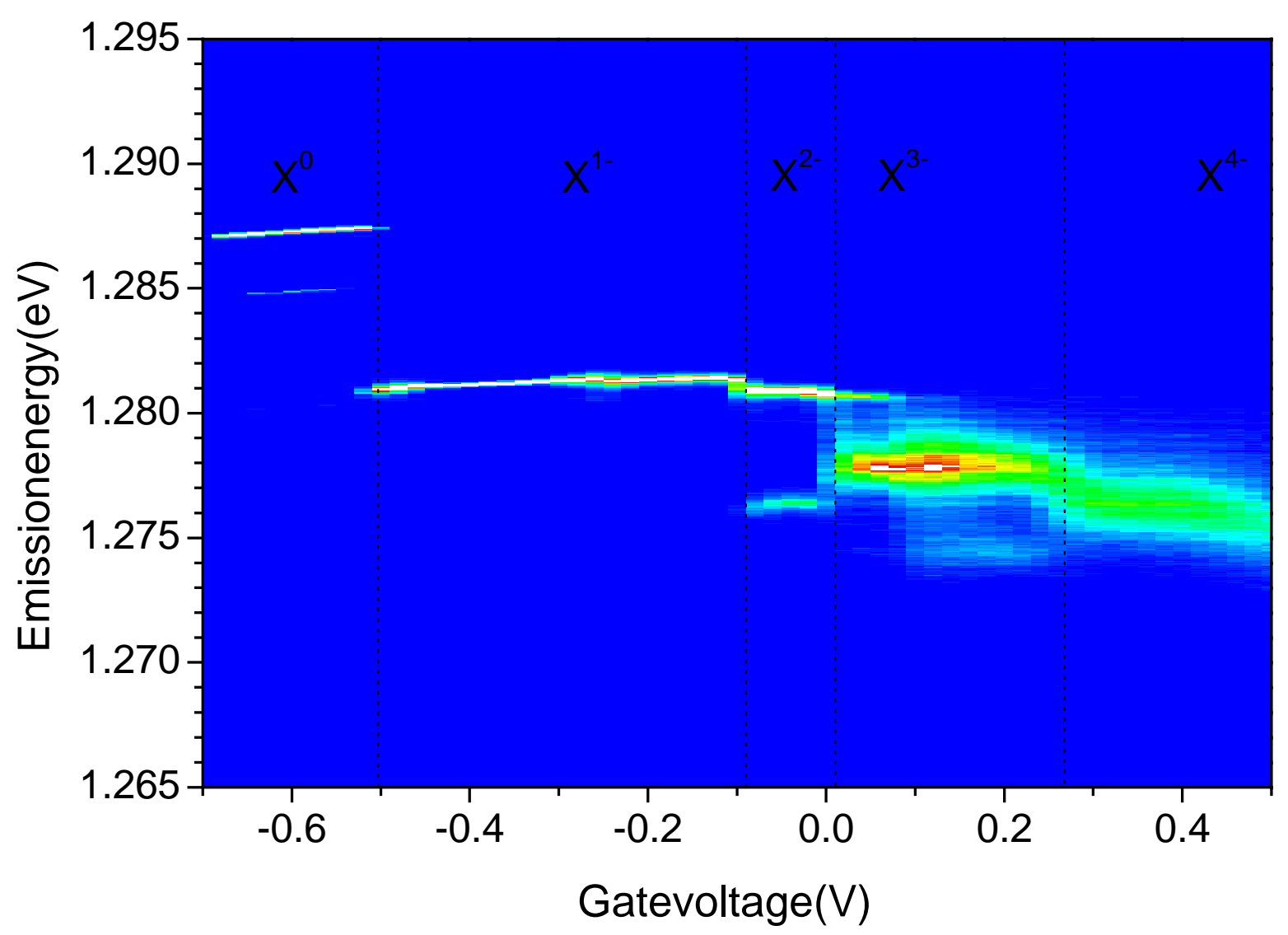

Figure 1: Emission energy versus gate voltage for an individual quantum dot at $4.2 \mathrm{~K}$. The PL is represented by a color scale: blue represents the background signal, yellow 450 and red 600 counts on the detector. The voltages at which the charge state of the exciton changes are marked, and the excitons are labelled $\mathrm{X}^{n-}$ where $n$ is the excess charge.

the $S=0$ state is broad with a line width of typically $\sim 0.5 \mathrm{meV}$. The reason for this is a spin-dependent energy broadening of the final state. The final state of the $\mathrm{X}^{2-}$ is metastable: at some point in time, the $p$ electron will relax and occupy the vacancy in the $s$ orbital. For the $S=0$ final state, this relaxation can occur without a spin flip, for instance by phonon emission, and this turns out to be a fast process, broadening the PL. The line width implies a relaxation time of $\sim 1$ ps. In contrast, for the $S=1$ final state, relaxation requires a spin flip, ruling out interaction with a phonon as a possible mechanism, and the sharp PL line in this case demonstrates that relaxation is hindered.

On close examination, the higher energy $\mathrm{X}^{2-} \mathrm{PL}$ is not a single line but a doublet (Fig. ). We find that the $\mathrm{X}^{2-} \mathrm{PL}$ has a characteristic signature with the lower energy component roughly twice as intense as its lower energy partner. This splitting results not from an interaction in the final state but from an interaction in the initial state (Fig. ). The interaction is the exchange interaction between the $p$ electron and the $s$ hole. For a neutral exciton, there is typically a dark $L=2$ exciton and a bright $L=1$ exciton, split in energy by the electron-hole exchange interaction [4]. For the $\mathrm{X}^{2-}$, there are also $L=2$ and $L=1$ states, 


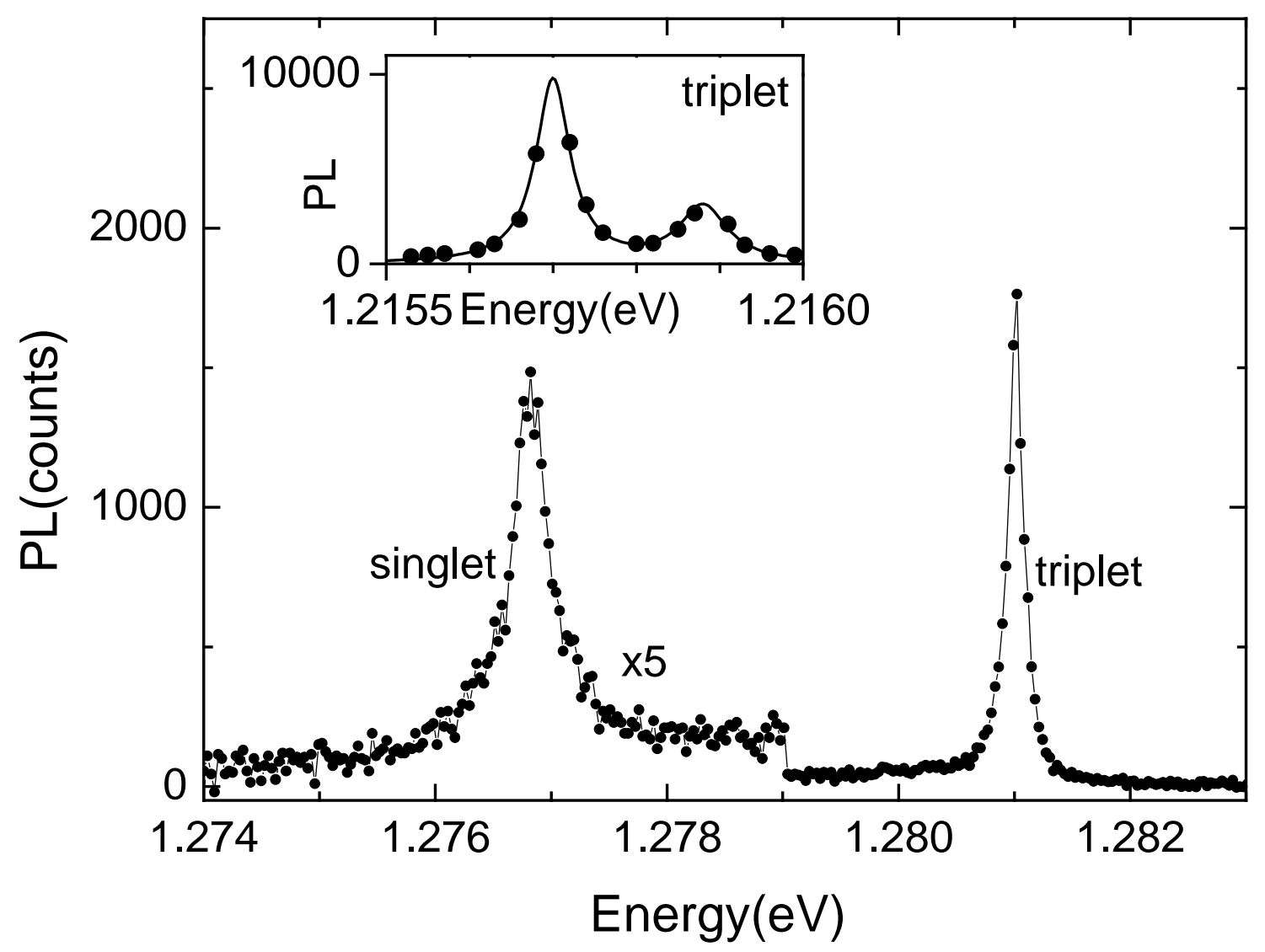

Figure 2: Emission from $\mathrm{X}^{2-}$ from an individual dot at $4.2 \mathrm{~K}$ labelled as triplet and singlet according to the spin of the final state. There is a hint of a splitting in the triplet emission; the inset shows the triplet emission for another dot where the triplet-singlet splitting increases to $8 \mathrm{meV}$. In this case, the triplet PL clearly consists of two lines. The solid line is a fit to two Lorentzians, the line width being determined by the spectral resolution of the spectrometer-detector system.

split by electron-hole exchange, but both are bright. The reason is that the $s$ hole has its exchange interaction with the $p$ electron but it recombines with an $s$ electron. The electron $s$ orbital is doubly occupied, so that the hole, regardless of its polarization relative to the $p$ electron, can always find a partner for recombination. The $\mathrm{X}^{2-}$ splitting is an example of exciton fine-structure, but a nice feature is that the fine structure is revealed without the need to convert a dark state into a bright one by breaking the symmetry. Another nice feature is that the $\mathrm{X}^{2-} \mathrm{PL}$ reveals simultaneously electron-electron exchange and electron-hole exchange, through the triplet-singlet splitting and the triplet fine-structure, respectively. The electron-hole exchange is an order of magnitude smaller than the electron-electron exchange because of the fact that the band index allows a hole to be distinguished from an electron whereas electrons are strictly indistinguishable. However, the $\mathrm{X}^{2-}$ electron-hole exchange is two orders of magnitude larger than that in a bulk semiconductor, and this simply reflects the strong quantization in self-assembled quantum dots. The explanation for the different 


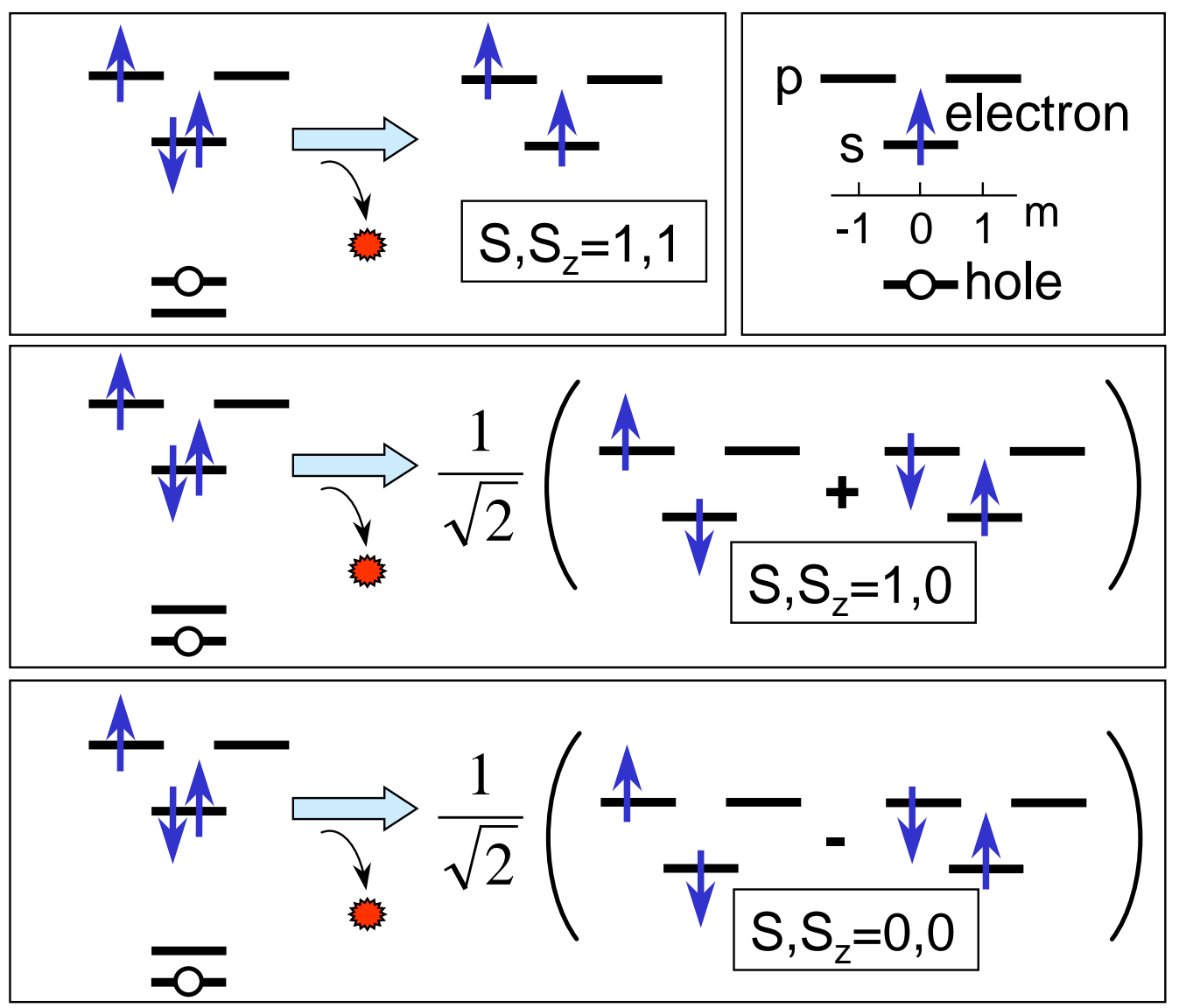

Figure 3: The configurations relevant to the $\mathrm{X}^{2-}$ emission process. Sketched are the electron $s$ and $p$ orbitals and the hole $s$ orbital. $m$ is the $z$-component of angular momentum. The electron spin is taken (arbitrarily) as up, and the electron-hole exchange interaction is illustrated by the (much exaggerated) splitting in the hole levels. The $S, S_{z}=1,1$ and 1,0 final states have the same energy, but the PL into these final states is split by the electronhole exchange interaction in the initial states (a few hundred $\mu \mathrm{eV}$ ). The $S, S_{z}=0,0$ final state lies above the $S=1$ states by twice the electron-electron $s-p$ exchange interaction (a few meV).

intensities in the fine structure is that the transition into the $S, S_{z}=1,1$ final state has the full oscillator strength whereas the oscillator strength for emission into the $S, S_{z}=1,0$ final state is reduced by a factor of two because of the admixture of an optically dark configuration (Fig. ).

\section{$S=1$ TRIPLY-CHARGED EXCITON}

The $\mathrm{X}^{3-}$ differs from the other excitons in that there are two possible initial configurations which lie within a few meV of each other. The $\mathrm{X}^{3-}$ has four electrons, two in the $s$ orbital and two in the $p$ orbitals. For a symmetric dot where the two $p$ orbitals are degenerate, each $p$ orbital is singly occupied in the ground state, as this allows the spins to be parallel so that 
the energy is lowered through the exchange interaction. This corresponds to Hund's rule in atomic physics. The ground state has therefore $S=1$. If however the two $p$ orbitals are split by more than the exchange energy between two $p$ electrons then a configuration with $S=0$ is energetically favored. In this case, the lower $p$ orbital is doubly occupied, corresponding to a closed shell and $S=0$. The PL is very different depending on the initial configuration. We consider initially the $S=1$ case.

For the $S=1 \mathrm{X}^{3-}$, there are two possible final states, $S=\frac{3}{2}$ and $S=\frac{1}{2}$, which are split by the exchange interaction. By diagonalizing the matrix of the Coulomb interactions, it can be shown that the splitting now becomes three times the $s-p$ exchange energy. This corresponds very closely to the experiments: on going from $\mathrm{X}^{2-}$ to $\mathrm{X}^{3-}$, the splitting in the experiments increases, by a factor very close to 1.5 for deeply confined dots. As for the $\mathrm{X}^{2-}$, the high energy line is sharp, the low energy line broad, and this is expected from the relaxation of the final state, just as for $\mathrm{X}^{2-}$. The $S=\frac{1}{2}$ final state can relax without a spin flip and has therefore a broad PL; the $S=\frac{3}{2}$ final state requires a spin flip for relaxation and can therefore have a sharp PL.

As for the $\mathrm{X}^{2-}$, a close inspection of the higher energy PL reveals a fine structure (Fig. refX3-). In this case, the peak is split into three closely spaced lines. Again, the explanation lies in the electron-hole exchange interaction. The initial state has $S=1$ with $S_{z}=-1,0,1$ resulting in three possibilities for the electron-hole exchange interaction, and hence three PL peaks. The highest energy line corresponds to emission from the $S_{z}=1$ initial state to the $S_{z}=\frac{3}{2}$ final state and clearly has the full oscillator strength. The other lines are less intense because, as for the $\mathrm{X}^{2-}$ fine structure, there is an admixture in the final states of configurations which do not contribute to the oscillator strength. For $\mathrm{X}^{3-}$ this is slightly more involved than for $\mathrm{X}^{2-}$ because there are three admixed configurations, two of which are dark. We predict that the intensities should scale as $3: 2: 1$. Experimentally, this is borne out very convincingly. Averaging over many dots to reduce any systematic errors involved in the measurement of just one dot, we find $3: 2: 1$. The observation of a triplet with a characteristic $3: 2: 1$ intensity ratio is very convincing evidence that the exciton in this case is following Hund's rule and forming an $S=1$ state.

\section{$S=0$ TRIPLY-CHARGED EXCITON}

The $\mathrm{X}^{3-}$ has a $S=0$ ground state in the presence of strong enough symmetry breaking. We have induced a change from the $S=1$ to the $S=0$ ground state by applying a magnetic field along the crystal growth direction. The magnetic field $B$ induces a splitting between the $m=-1$ and $m=1 p$ orbitals; the splitting is equal to the cyclotron energy for a parabolic potential [2]. An example is shown in Fig. where the splitting characteristic of the $S=1$ $\mathrm{X}^{3-}$ "collapses" at around $1 \mathrm{~T}$. At this magnetic field, there is a transition from an $S=1$ to an $S=0$ initial state, shown diagrammatically in Fig. . It is natural that the "collapsed" PL lies in between the exchange split peaks of the $S=1 \mathrm{X}^{3-}$ because in the final state after photon emission, electron-electron exchange does not play a major role (Fig. ). The large PL line width of the $S=0 \mathrm{X}^{3-}$ is related to the fast relaxation of the final state, as for the $\mathrm{X}^{2-}$ singlet peak, but, as we show below, there is an important additional factor.

We find that we can also force the change in $\mathrm{X}^{3-}$ ground state from $S=1$ to $S=0$ with the gate voltage, the dot in Fig. being a clear example. For this dot, the $\mathrm{X}^{3-}$ forms at a voltage, $0.01 \mathrm{~V}$, when some charge occupies not just the dots but also the wetting layer, the 


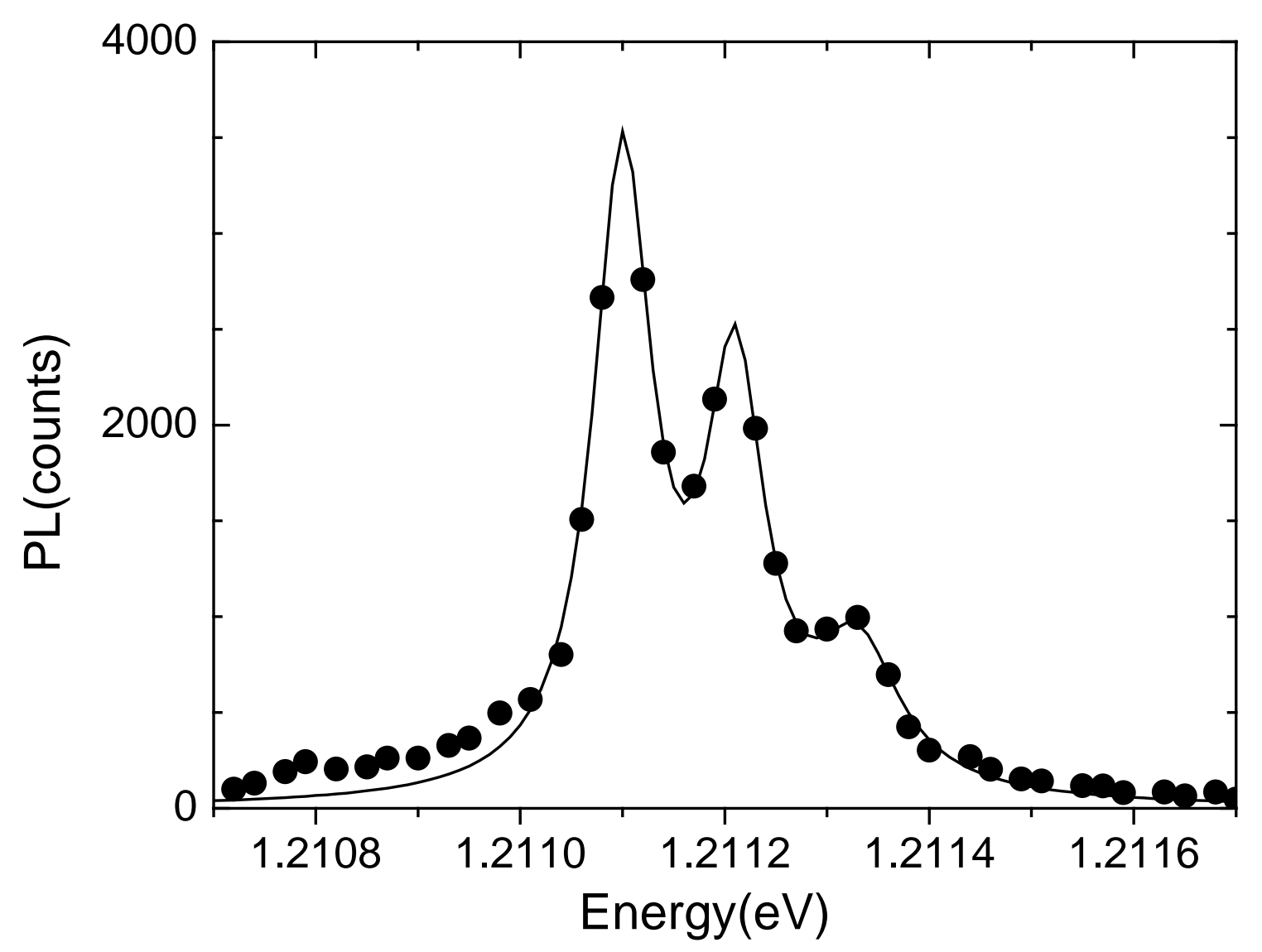

Figure 4: Fine structure of the PL from an $\mathrm{X}^{3-}$ showing emission into the $S=\frac{3}{2}$ final state. The points are the measured data; the solid line is a fit to three Lorentzians. As in Fig. 2, the lines are not completely resolved.

thin InAs layer connecting the quantum dots. We can verify this by magneto-capacitance measurements (not shown here). It can be seen in Fig. that the $\mathrm{X}^{3-}$ lies in between the exchange-split $\mathrm{X}^{2-}$ lines, and that the $\mathrm{X}^{3-}$ does not have a splitting. It has therefore "collapsed". In fact, we can prove that the peak at $V \geq 0.01 \mathrm{~V}$ is indeed $\mathrm{X}^{3-}$ recombination by increasing the temperature slightly, allowing us to populate thermally also the $S=1$ $\mathrm{X}^{3-}$. Two peaks appear in the spectrum with line widths and a splitting characteristic to the $S=1 \mathrm{X}^{3-}$. The mechanism which favors the $S=0$ over the $S=1 \mathrm{X}^{3-}$ ground state when the wetting layer is occupied is not known. It might be a consequence of a slight anisotropy in the wetting layer continuum, which, through an electrostatic interaction, lifts the near-degeneracy of the $p$ orbitals.

\section{BEYOND THE ARTIFICIAL ATOM MODEL}

A new type of configuration mixing arises when the $\mathrm{X}^{3-}$ forms the $S=0$ state. In the final state after photon emission, the lower $p$ orbital is doubly occupied, and there is a vacancy in the $s$ orbital. The good quantum numbers, angular momentum and spin, can be preserved by promoting one of the $p$ electrons to an unoccupied $d$ orbital, with the other $p$ electron 


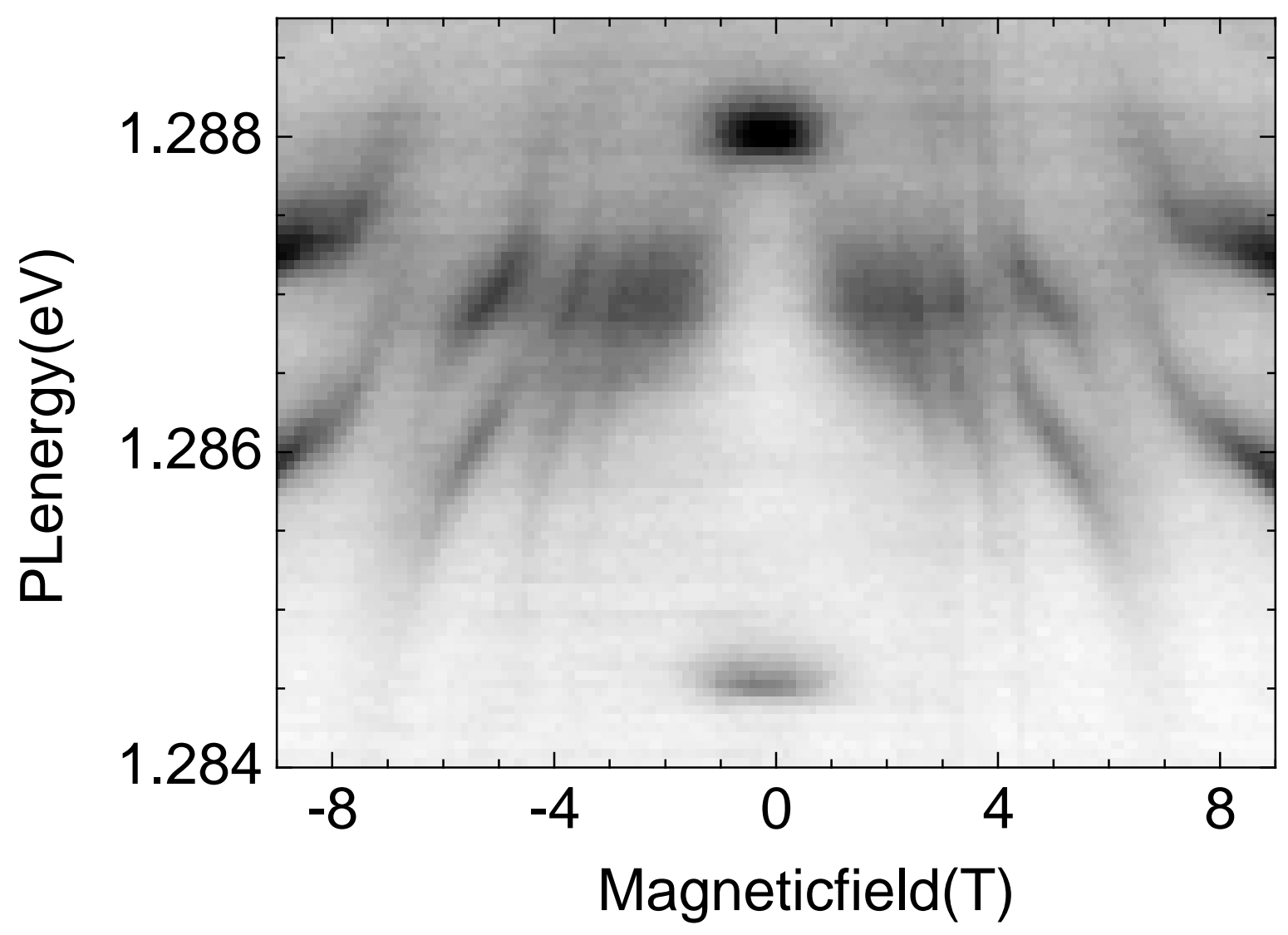

Figure 5: A grey-scale plot of the PL from an $\mathrm{X}^{3-}$ as a function of magnetic field. At $B=0$, there are two PL lines, the higher (lower) energy line is emission into the $S=\frac{3}{2}\left(S=\frac{1}{2}\right)$ final state, split by three times the $s-p$ exchange. At $B \sim 1 \mathrm{~T}$, the initial state changes from $S=1$ to $S=0$.

filling the vacancy in the $s$ orbital, as illustrated in Fig. . The resulting configuration has a very similar energy to the first, and therefore the two configurations are admixed in the final state wave function. This means that the PL is influenced by the $d$ orbital even though the $d$ orbital is not occupied in the initial state. For our dots, the $d$ orbitals are not bound; instead, there are continuum states at higher energy. This means that continuum states are admixed into the final state of the $S=0 \mathrm{X}^{3-}$ wave function (Fig. ). This contributes to the broadening of the collapsed $\mathrm{X}^{3-}$, either in a magnetic field or with an occupied wetting layer, because it allows the final state to decay very rapidly. We emphasize that this process is forbidden for the $S=1 \mathrm{X}^{3-}$.

The consequences of the hybridization with continuum states are particularly pronounced in a magnetic field when the continuum density of states condenses into a series of Landau levels. A resonance with the $n$th Landau level $(n=0,1,2 \ldots)$ occurs when the separation between the dot $p$ and $s$ orbitals equals the separation between the $n$th Landau level and the $p$ orbital (Fig. ). Assuming a symmetric, harmonic oscillator model, the separation between the $p$ and $s$ levels decreases by $\frac{1}{2} \hbar \omega_{c}$ with magnetic field, where $\omega_{c}=e B / m^{*}$ is the cyclotron 


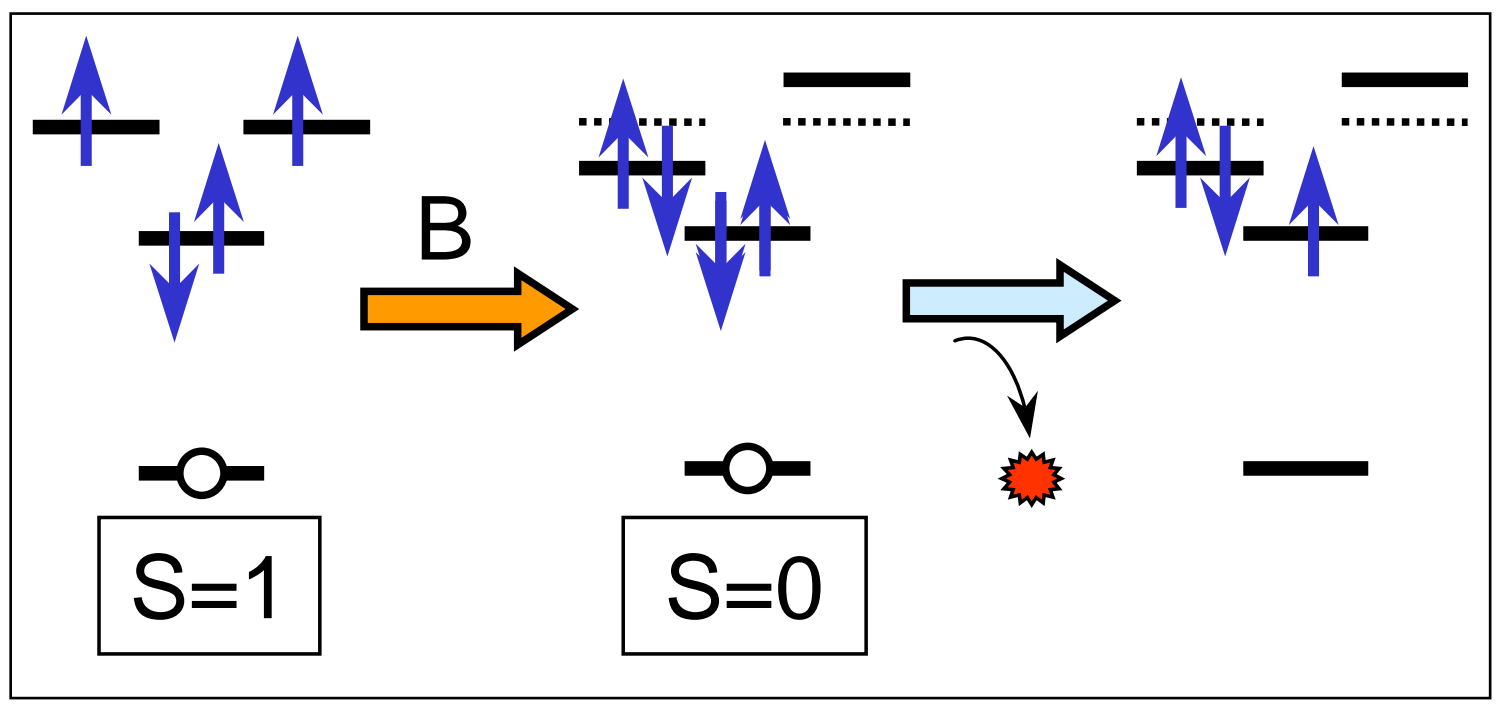

Figure 6: The $\mathrm{X}^{3-}$ configurations (ignoring states at higher energy than the $s$ and $p$ orbitals). At $B=0$ (left), the initial state has lowest energy with $S=1$. A magnetic field lifts the degeneracy between the two $p$ orbitals such that at a critical magnetic field $(\sim 1 \mathrm{~T}$ for the dots investigated here) the ground state has $S=0$ with both electrons occupying the lower $p$ orbital. The final state after photon emission is shown in this approximation.

energy $\left(m^{*}\right.$ is the effective mass). This leads to the resonance condition $\left(n+\frac{3}{2}\right) \hbar \omega_{c}=\Delta$ where $\Delta$ is the excess kinetic energy at $B=0$ (see Fig. ). This analysis predicts a series of oscillations, periodic in $1 / B$, and this is exactly what we observe, as shown in the PL spectra in Fig. . Furthermore, we identify the resonances with the fields at which there is a pronounced anti-crossing in the PL, and this gives very convincing agreement with our resonance condition, shown graphically in Fig. ). Taking $m^{*}=0.07 m_{o}$, we find $\Delta \simeq 20 \mathrm{meV}$. This value is much larger than all the shifts and splittings on charging, and must therefore be related to a quantization energy. This is accounted for quite naturally in the Auger-like process we propose. In the light of this analysis, the oscillation at $7 \mathrm{~T}$ in Fig. refX3-B is with the $n=0$ Landau level, and is therefore the last resonance. At higher field, the Landau levels are too distant in energy for a strong hybridization with the dot levels to take place. In other words, at intermediate magnetic fields, the final state of the $\mathrm{X}^{3-}$ is a very unusual hybridization of localized quantum dot and delocalized Landau level states [5], but at very large magnetic field, the hybridization is turned off and the dot behaves again as an artificial atom.

In addition to the hybridization of the final state, we have also evidence for hybridization of the initial state with the continuum. In the regime where the wetting layer is occupied, the dot in Fig. exhibits a transition from $\mathrm{X}^{3-}$ to $\mathrm{X}^{4-}$. It can be seem that the PL moves smoothly from one exciton energy to the other. In stark contrast, at negative voltages where the wetting layer is unoccupied, there is never a measurable PL signal in between the PL lines in the transition regimes from one charge state to the next. We believe that the $\mathrm{X}^{3-}$ to $\mathrm{X}^{4-}$ transition in Fig. represents a hybridization of the dot with the Fermi energy of the 


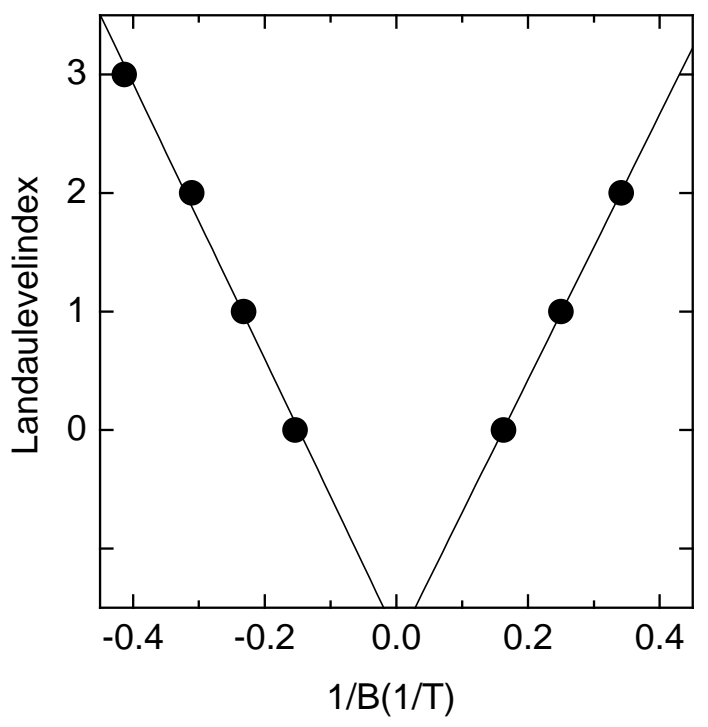

Figure 7: The inverse magnetic fields at which the PL in Fig. 5 exhibits pronounced anticrossings plotted against Landau index $n$ both for positive and negative $B$. The solid lines are straight line fits to the data.

two-dimensional electron gas in the Fermi energy such that the average charge on the dot changes gradually with increasing voltage [5]. Strong support for this idea comes from a measurement of $d E_{f} / d V_{g}$, the rate of change of the Fermi energy $E_{f}$ with the gate voltage, which we determine to be $17 \mathrm{meV} / \mathrm{V}$ from the magneto-capacitance experiment. We also calculate exactly this value from the electrostatics of our device [5] Experimentally, the PL transition from $\mathrm{X}^{3-}$ to $\mathrm{X}^{4-}$ moves with $-15 \pm 5 \mathrm{meV} / \mathrm{V}$, offering strong support to our assertion that the exciton is coupled to the Fermi energy of the two-dimensional electron gas associated with the wetting layer.

\section{CONCLUSIONS}

The optical emission from a charge-tunable quantum dot reveals. We find red-shifts on charging, particularly pronounced on going from the neutral exciton to the singly charged exciton, an optical manifestation of a shell structure. The doubly and triply charged excitons exhibit splittings which are related to exchange effects, both electron-electron and electronhole interactions. We show that the initial state configuration of the triply charged exciton can be changed from spin 1 to spin 0 either by a magnetic field or by loading the wetting layer with electrons, and we argue that this change in spin introduces a new possibility for configuration mixing: in the final state, higher energy states are inevitably admixed into the wave function. This should be a common feature of more-highly charged excitons. In our present dots, this process leads to a pronounced hybridization with the continuum.

This work was funded by EPSRC and the DFG (SFB348). 

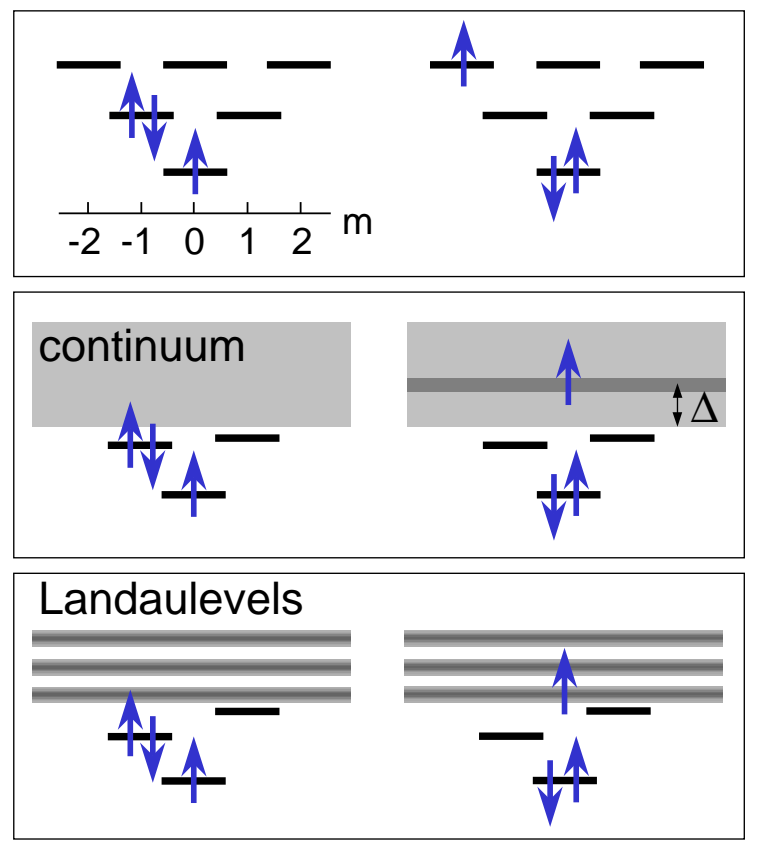

Figure 8: Top: The new possibility for configuration mixing which arises for the final state of the $S=0 \mathrm{X}^{3-}$. Both configurations have the same quantum numbers and are admixed. This is the situation for a deep quantum dot with confined $d$ orbitals. If the $d$ states are unbound (middle panel), an electron is promoted into the continuum. In a magnetic field (bottom panel) there are resonances with the Landau levels. The situation shown corresponds to that in the experiments at $B=7 \mathrm{~T}$ where there is a resonance with the $n=0$ Landau level. The energy $\Delta$ is determined by the frequency of the oscillations in $1 / B$. For the dot in Fig. 5, $\Delta \simeq 20 \mathrm{meV}$.

\section{References}

[1] G. Bastard, Wave mechanics applied to semiconductor hetersostructures (Wiley, 1988).

[2] R. J. Warburton, B. T. Miller, C. S. Dürr, C. Bödefeld, K. Karrai, J. P. Kotthaus, G. Medeiros-Ribeiro, P. M. Petroff, and S. Huant Phys. Rev. B 58, 16221 (1998).

[3] R. J. Warburton, C. Schäflein, D. Haft, F. Bickel, A. Lorke, K. Karrai, J. M. Garcia, W. Schoenfeld, and P. M. Petroff, Nature (London) 405, 926 (2000).

[4] M. Bayer, G. Ortner, O. Stern, A. Kuther, A. A. Gorbunov, A. Forchel, P. Hawrylak, S. Fafard, K. Hinzer, T. L. Reinecke, S. N. Walck, J. P. Reithmaier, F. Klopf, and F. Schäfer, Phys. Rev. B 65, 195315 (2002).

[5] A. O. Govorov, K. Karrai, R. J. Warburton, and Kalameitsev, in the proceedings of this conference. 